\title{
THE GREEN AGGREGATES FOR SUSTAINABLE DEVELOPMENT IN CONSTRUCTION INDUSTRY
}

\author{
Sundeep Chowdary Daggubati ${ }^{1}$, Nazneen ${ }^{2}$, Subham Sharma $^{3}$, Sulabh Raj Gurung ${ }^{4}$ \\ ${ }^{1}$ Final year, Bachelor of Engineering, Department of Civil Engineering, Hindustan Institute of Technology \& Science, \\ Chennai, India \\ ${ }^{2}$ Final year, Bachelor of Engineering, Department of Civil Engineering, Hindustan Institute of Technology \& Science, \\ Chennai, India \\ ${ }^{3}$ Final year, Bachelor of Engineering, Department of Civil Engineering, Hindustan Institute of Technology \& Science, \\ Chennai, India \\ ${ }^{4}$ Final year, Bachelor of Engineering, Department of Civil Engineering, Hindustan Institute of Technology \& Science, \\ Chennai, India
}

\begin{abstract}
Solid waste management is being done in several ways but there is a need for an effective and a creative way that aims to bring sustainable development in the construction industry. Existing methods of waste management and disposal like the land filling is ultimately polluting the soil and ground water. The need for manufactured aggregates is to promote the $3 R$ 's (Reduce, Reuse \& Recycle)and to achieve a sustainable development. Manufactured aggregatesmay reduce environmental strain by minimizing over exploitation and indiscriminative use of natural aggregates which we get from the mining, quarrying and blasting activities. Waste is not a waste unless you waste it. This paper focuses on the synthesis of an ingenious construction material (Coarse aggregates) from the various industrial wastes which are ceramic materials, lathe waste and plastics (Low density \& Medium density polyethylene) along with slag (fly ash/Ground Granulated Blast furnace Slag) in definite proportions. Coarse aggregate synthesised through this process have both advantages and disadvantages when compared to the natural aggregates. Various tests for aggregate which are specified in the IS 2386 does not seem applicable as theseartificial aggregates undergo elastic or plastic deformation.
\end{abstract}

Keywords: Aggregates, Ceramics, Industrial waste, Lathe waste, Plastics, Quarry dust.

\section{INTRODUCTION}

Over exploitation of resources is what the human being got habituated to do with the environment. Indiscriminate usage and exploitation of resources is causing environmental strain. Mining and quarrying for the natural aggregates which are prerequisites for every construction activity is depleting the natural resources and deteriorating the environment(GuttilaYuganthaJayasinghe et al.) [1]. Activities like mining and quarrying are resulting in the loss of habitat, agricultural lands, forests, Bio-diversity and are also resulting undesirable climatic changes. Mining activities and stone crushing units contribute to both sound and air pollution. The current years are already witnessing the scenario of fast depletion of natural resources and the construction industry that mostly depends on aggregates is also on the verge of loss due to their shortage and unavailability.Natural aggregates still remain as the most mined material in the world (Tor Arne Hammer et al.) [2]. Environmental laws and restrictions are curbing the mining activities as far as possible.River sand mining is almost banned in India and there won't be any future contracts given by the government to private mining agencies. Recent decades have seen a marked upsurge in industrial and economic growth, contributing to an improved quality of life and well-being for citizens (Andrés Juan et al.) [3].
However, we should not lose sight of the fact that every production system creates by-products and waste products which can affect the environment. These effects may occur at any point in the product's life-cycle, whether during the initial phase of obtaining raw materials, during the transformation and production phase, during product distribution or when the end user must dispose of products which are no longer required.As a result, recent years have witnessed rising social concern about the problem of waste management in general, and industrial waste and waste from the construction industry in particular. This problem is becoming increasingly acute due to the growing quantity of industrial, construction and demolition waste generated despite the measures which have been taken in recent years.

\section{MATERIAL SELECTION AND} COMPOSITION

Artificial aggregates were assumed to be formed from the combination of a binder and ingredients which are industrial wastes. The binder needs to be a pollutant which is a liquid or semi-solid or amorphous substance, industrial waste, should be non-reactive (inert) and should have the ability to hold the ingredients (solid wastes) intact with each other to form a heterogeneous mixture that can be used in casting 
aggregates.The following materials are chosen based on their following characteristics which they exhibit when alone or in combination:Ceramics- Breakage or deformation, which does not affect the intrinsic characteristics of the Ceramic material; Reduced $\mathrm{CO}_{2}$ emissions; Long-term mechanical strength; Stable resistance to expansion due to the presence of free lime, sulphates andaggregate-alkali reactions;Durability and resistance to the action of pure and acid water; Reduced hydration heat; Impermeable; Reduced porosity and increasedcompactness.Slag (DarkoKokot et al. \&SanjaDimter et al.) [4-5]- Excellent mechanical properties; Inertness to chemicals; Impermeable; Mouldable; Adhesion.Plastics- Reduces water absorption; reduced porosity; Binding and lamination.Lathe waste- Lightweight; Supplements strength; Reinforces the mixture.

\section{PROPORTIONATING}

Manufactured aggregates constituted equal volumes of solid wastes which included ceramic pieces of size less than 12.5 $\mathrm{mm}$ and greater than $10 \mathrm{~mm}$, crushed lathe waste, amorphous waste including mostly fly ash and quarry dust. Plastic chunks which were used to hold the ingredients of at least 4 to 5 manufactured aggregates are of size $3 \mathrm{~cm} \times 5 \mathrm{~cm}$ and are 2 in number. These are overlapped on each other allowing space for solid wastes to get sandwiched in between them. The number of such plastic chunks that were softened to hold the solid wastes may vary according to the type of plastic being employed in binding. Several trials were conducted to establish the required number of the quantity of plastic waste needed for the purpose of softening and binding the solid wastes.
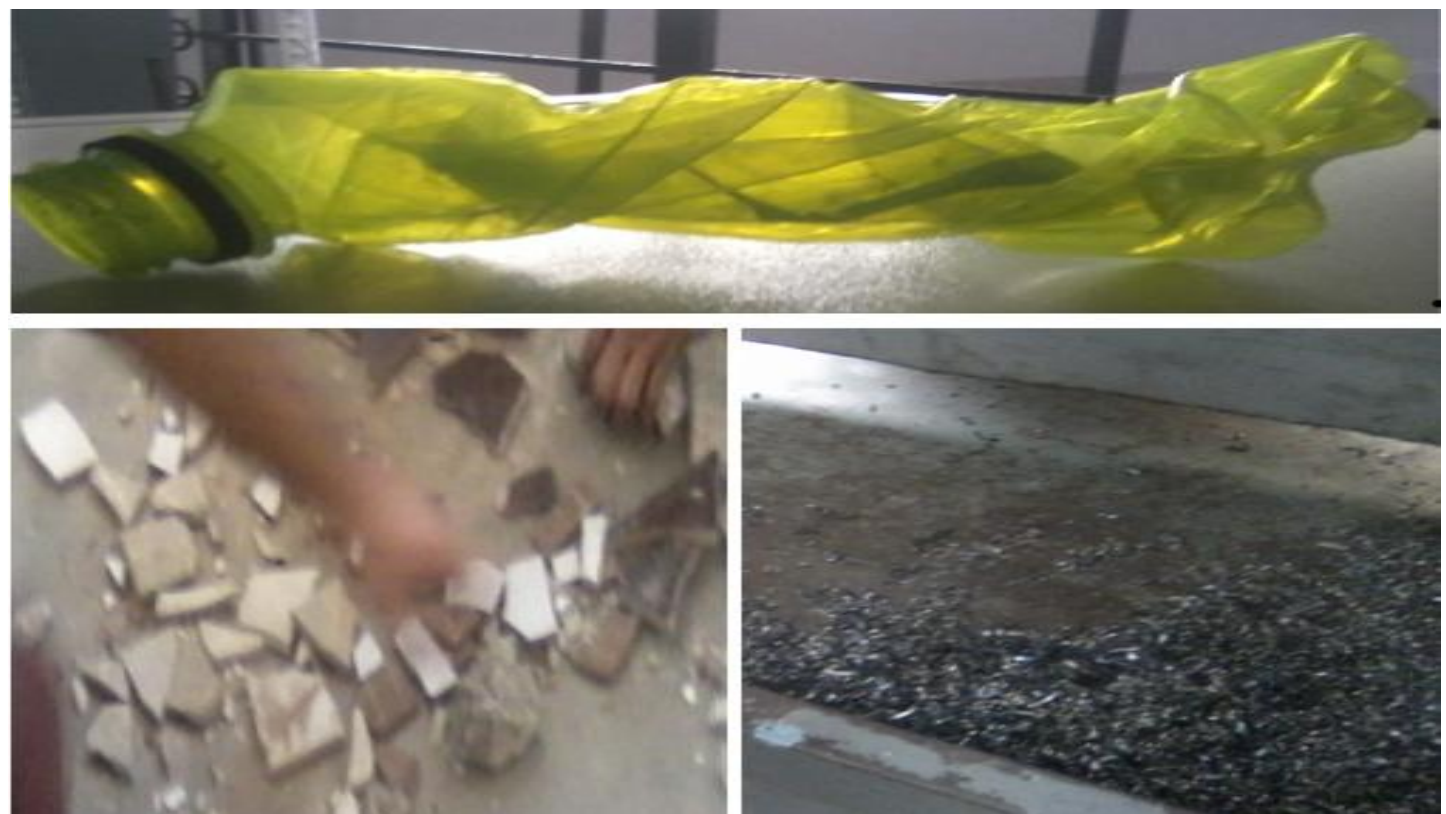

Fig 1 Constituents of manufactured aggregates-PET bottle, Ceramic waste \& Lathe waste.

\section{MOLTEN PLASTIC AS A BINDER}

Medium density polyethylene products like the Polyethylene terephthalate bottles and Low density polythene carry bags were chosen and were softened to use the plastic matrix formed by them to impregnate the solid wastes. In this process, Size and shape of the aggregates is mainly dependent upon the mould or the container used in the muffle furnace. Muffle furnace should be operated between 350 to 375 degree Celsius to ensure uniform lamination and bonding of plastic with the solid wastes (ingredients). Aggregates are cooled down to the normal room temperature by taking them out just after placing them in the muffle furnace for 35 to 40 seconds.
Large scale production is also possible through a customised machinery and a plant that would allow the following operations to take place: Collection and segregation of waste, grading of waste, Plastic softening equipment, temperature controlled chamber to let the molten plastic flow, proportionating unit, moulders and a cooling tank. A large scale setup is both feasible and viable as the resources are cheaply available and are huge in terms of quantity. Procuring resources from distant and scattered areas may affect the logistics and could become a risk if not properly planned and analysed. In addition to attaining proximity to resources there are risks which are related to segregation of plastic wastes, meeting the required specifications and making the material cost effective.

Table 1Cost of resources

\begin{tabular}{|l|l|l|}
\hline Material & Quantity & Cost * \\
\hline $\begin{array}{l}\text { Ceramic Waste } \\
\text { (Tiles , crockery, bathroom }\end{array}$ & $15 \mathrm{~kg}-20 \mathrm{~kg} \mathrm{sack}$ & Rs 40/- \\
\hline
\end{tabular}




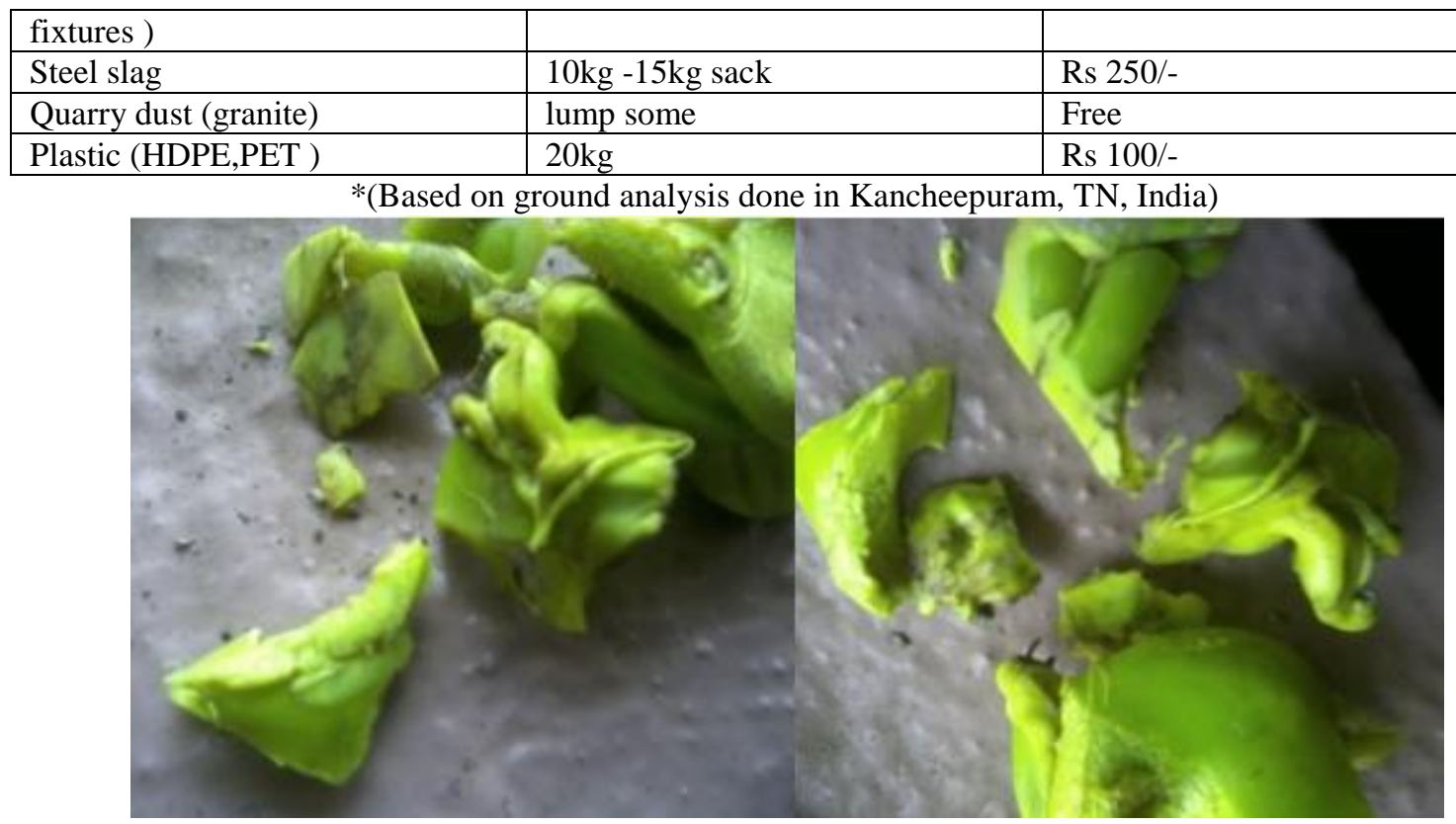

Fig 2 Manufactured aggregates using plastic as a binder.

\subsection{Disadvantages of Plastic Matrix as a Binder}

Mass production of these aggregates seemed impossible with the available laboratory equipment as it needs a customised machinery. Number of aggregates (Coarse) that can be synthesised per one medium sized PET bottle were only 3 to 5 . Controlled softening of plastic and uniform coating or lamination seemed tough to achieve using the laboratory equipment. Volatile Organic Compounds emission is another drawback.

\subsection{Advantages of Plastic Matrix as a Binder}

Besides these drawbacks encountered during the synthesis of artificial aggregates with plastic as a binder, Plastic as a binder gave very light weight aggregates which are absolutely hydrophobic, highly inert and coloured aggregates. Aggregates obtained were angular and some are rounded too with grooves on them which may facilitate good interlocking. Aggregates are not brittle and they underwent plastic deformation under crushing and impact loads because of which tests specified in IS: 2386-1963 [6] seemed unsuitable.

\section{EXPERIMENTAL STUDY}

Most of the standard and important tests for aggregates like the aggregate impact, crushing and abrasion could not be conducted as these manufactured aggregates did not undergo changes which the natural aggregates will undergo when load is applied on them. To determine these important results which are mainly related to the strength characteristics of the manufactured aggregates, existing code of practice for the aggregate tests may need to be revised. Aggregates were primarily tested to assess their suitability for the application as road aggregates so their compatibility with concrete is yet to be checked and known. The following tabulation shows the results of various tests conducted on the manufactured aggregates:

Table 2 Summary of experimental investigation conducted on manufactured aggregates.

\begin{tabular}{|l|l|l|}
\hline Test & Value & Remarks \\
\hline Water absorption & $0.17 \%$ & Low \\
\hline Specific gravity & 2.8 & Average \\
\hline Light weight & $480 \mathrm{~g}$ less per Kg & Very Light weight \\
\hline $\begin{array}{l}\text { Soundness } \\
\text { Leaching with Sodium sulphate } \\
\text { Leaching with magnesium sulphate }\end{array}$ & Could not be determined. & $\begin{array}{l}\text { No } \\
\text { witnessed. Inert. }\end{array}$ \\
\hline Aggregate crushing value & Could not be determined. & $\begin{array}{l}\text { Plastic } \\
\text { occurred. Not brittle. }\end{array}$ \\
\hline Los Angeles abrasion value & Could not be determined. & Hard. \\
\hline Angularity number & 7 & Not flaky \\
\hline $\begin{array}{l}\text { Shape } \\
\text { Flakiness Index } \\
\text { Elongation Index }\end{array}$ & $18.22 \%$ & $\begin{array}{l}\text { Angular } \\
\text { Angular }\end{array}$ \\
\hline Stripping value & $16 \%$ & Good adhesion \\
\hline
\end{tabular}




\begin{tabular}{|l|l|l|}
\hline & & bitumen. \\
\hline Bulk density & $1087 \mathrm{Kg} / \mathrm{m}^{3}$ & Light weight/ more voids \\
\hline pH & 8.7 & Slightly alkaline \\
\hline
\end{tabular}

\section{CONCLUSIONS}

As far as the consumption of natural aggregates is concerned, the use of artificial and recycled aggregates as a partial replacement of natural ones has to be encouraged (Priyadharshini et al.) [7]. In this context, the recycling of huge amounts of industrial solid wastes, sludge, reservoir sediments and demolition wastes in the production of concrete and aggregates is an important issue in directing waste management towards a more sustainable development. In addition to helping protect the environment, use of such waste offers a series of advantages such as a reduction in the use of other raw materials, contributing to an economy of natural resources. Moreover, reuse also offers benefits in terms of energy, primarily when the waste is from kiln industries (the ceramics industry) where highly endothermic decomposition reactions have already taken place, thus recovering the energy previously incorporated during production (Claudio Ferone et al.) [8]. Reusing plastics to manufacture various items of commercial use other than manufacturing aggregates as proposed may give rise to inferior products that ultimately end up as wastes. Hence, there is a scope for managing plastic wastes effectively through the manufacturing of aggregates. Mining and quarrying can be reduced by employing alternative materials like these. The manufactured aggregates may become substitutes to the naturally aggregates when they become scarce in the near future. Utilisation of these aggregates for non-structural applications ensures sustainable development in the construction industry.

\section{REFERENCES}

[1]. GuttilaYuganthaJayasinghe and Yoshihiro Tokashiki, Synthetic Aggregates Produced by Different Wastes as a Soil Ameliorant, a Potting Media Component and a Waste Management Option, Source: Waste Management, Book edited by: Er Sunil Kumar, ISBN 978-953-7619-84-8, pp. 232, March 2010, INTECH, Croatia, downloaded from SCIYO.COM

[2]. Tor Arne Hammer, SINTEF Building and Infrastructure, Concrete, Trondheim, Norway, Source: Internet document [3]. Andrés Juan, César Medina, M. Ignacio Guerra, Julia $\mathrm{M}^{\mathrm{a}}$ Morán, Pedro J. Aguado, $\mathrm{M}^{\mathrm{a}}$ Isabel Sánchez de Rojas, MoisésFrías and Olga Rodríguez, Re-use of ceramic wastes in construction, Source-wwww.intechpoint.com.

[4]. DarkoKokot, ZAG, ZvonkoCotič, Primorjed.d., Loris Bianco, Ferriere Nord, S.p.A., Safwat Said, VTI, Application of steel slag aggregate in road construction, Online document.

[5]. Ivana Barišić, SanjaDimter, IvankaNetinger, Possibilities of application of slag in road construction, ISSN 1330-3651, Technical Gazette 17, 4(2010), 523-528. [6]. IS 2383-1963 Part 1 to 5, Bureau of Indian Standards 2007.
[7]. Priyadharshini, Mohan Ganesh.G, Santhi.A.S, Experimental study on Cold Bonded Fly Ash Aggregates, ISSN 0976 - 4399, International journal of civil and structural engineering.

[8]. Claudio Ferone 1,*, Francesco Colangelo 1, Francesco Messina, Coal Combustion Wastes Reuse in Low Energy Artificial Aggregates Manufacturing, ISSN 1996-1944, www.mdpi.com/journal/materials 